\title{
Feature and Label Relation Modeling for Multiple-Facial Action Unit Classification and Intensity Estimation
}

\author{
Shangfei Wang ${ }^{\mathrm{a}, *}$, Jiajia Yang ${ }^{\mathrm{a}}$, Zhen $\mathrm{Gao}^{\mathrm{a}}$, Qiang $\mathrm{Ji}^{\mathrm{b}}$ \\ ${ }^{a}$ Key Lab of Computing and Communication Software of Anhui Province \\ School of Computer Science and Technology, University of Science and Technology of China \\ Hefei, Anhui, P.R.China, 230027 \\ ${ }^{b}$ Department of Electrical, Computer, and Systems Engineering, Rensselaer Polytechnic Institute
} Troy, NY, USA, 12180

\begin{abstract}
In this paper, we propose multiple facial Action Unit (AU) recognition and intensity estimation by modeling their relations in both feature and label spaces. First, a multi-task feature learning method is adopted to learn the shared features among the group of facial action units, and recognize or estimate their intensity simultaneously. Second, a Bayesian network is used to model the co-existent and mutualexclusive semantic relations among action units. Finally, through probabilistic inference, the learned Bayesian network combines the results of the multi-task learning with the AU relations it captures to perform multiple AU recognition and AU intensity estimation. Experiments on the extended Cohn-Kanade database, the MMI database, the McMaster database and the DISFA database demonstrate the effectiveness of our method for both AU classification and AU intensity estimation.
\end{abstract}

Keywords: AU recognition; AU intensity estimation; AU relation modeling; Multi-task feature learning; Bayesian network

\footnotetext{
*This is the corresponding author (Telephone: +86-0551-63602824).

Email addresses: sfwang@ustc . edu . cn (Shangfei Wang), yang25@mail . ustc. edu. cn (Jiajia Yang), gzgql l xh@mail . ustc.edu. cn (Zhen Gao), qji@ecse.rpi.edu (Qiang Ji)
} 


\section{Introduction}

Recent years have seen an increasing attention and considerable progress on facial Action Unit (AU) analysis due to its wide applications in human-computer interaction [1]. The main stream of current $\mathrm{AU}$ analysis either recognizes each AU individually or recognizes the fixed AU combinations. They thus either ignore the dependences among multiple AUs, or cannot handle thousands of possible combinations. Only recently, several work exploits AU dependencies to facilitate AU analyses from target labels or image features. However, little work leverages the relations embedded in both AU labels and image features for AU analyses. Since several AUs can be present at the same image, the dependencies inherent in target labels and in the shared features among multiple AUs carry crucial top-down and bottom up evidence respectively for improving AU analysis.

Therefore, in this paper, we tackle the problem of AU recognition and AU intensity estimation by exploiting the relations of AUs from both shared features and target labels. First, a multi-task learning (MTL) algorithm is adopted to learn the shared features among AUs and recognize multiple AUs or estimate multiple AU intensity simultaneously. Second, a Bayesian network (BN) is used to model AUs' relations from labels by structure and parameter learning. Finally, the outputs of multi-task learning algorithm are used as the inputs of the learned $\mathrm{BN}$ to obtain improved multiple AU recognition and intensity estimation. Experimental results on the extended Cohn-Kanade (CK+) database and the MMI database demonstrate that MTL outperforms single task learning, and the relationship model from AU labels further improves the performance of AU classification and the crossdatabase experiment shows the generalization ability of our relationship model. The results on the McMaster databases and the DISFA database for AU intensity estimation also indicate that learning the shared feature in each AU group by MTL improve the performance by single task learning and our BN model modeling the AU relationship further improve the AU prediction result by MTL.

The paper is organized as follows: Section 2 briefly reviews the related works on AU analysis. Section 3 describes the details of our proposed AU classification and AU intensity estimation approach considering the relations among AUs from both features and labels. Section 4 provides the experiments and analyses on four benchmark databases. Section 5 summarizes our work briefly. 


\section{2. related work}

Usually, several AUs can be present at the same image or an image sequence. Thus, AU recognition can be formulated as a multi-label problem. Due to the large number of possible label sets, multi-label recognition is rather challenging. Successfully exploiting the dependencies inherent in multiple labels is the key to facilitate the learning process. Present AU recognition research can be divided into three groups.

The first group recognizes each AU individually [2][3] directly from images or sequences. They are referred to as image-based AU recognition methods. For example, Valstar and Pantic [2] detected and tracked 20 facial points, and then used a combination of gentleBoost, support vector machines, and hidden Markov models as a classifier to detect 22 AUs [2]. Maaten et.al [3] adopted Active Appearance Model (AAM) features and linear chain conditional random field for AU recognition. These works treat each AU recognition individually as one-vs.-all scheme, do not consider the AUs' relations existing in features or labels. However, multiple AUs can appear together, and there exist dependencies among them. Exploiting such dependencies may help AU recognition and modeling.

The second group recognizes AU combinations. Littlewort et al. [4] adopted a linear SVM with Gabor features to analyze the AU combinations of $1+2,2+4$, $1+4$, and 1+2+4. Lucey et al. [5] detected a few combinations of AUs (i.e. 1, $1+2,4,5)$ using SVM and Nearest Neighbor with AAM features. Although the co-existent relations among AUs in an AU combination has been exploited by the used features and classifier in these works, the combinations are manually determined and fixed. Each combination is regarded as a new AU. Thus, it is only feasible for a few combinations, and hard to detect thousands of possible combinations. In addition, such AU combinations only capture coexistent AUs. They cannot capture AUs that are mutually exclusive of each other.

The third group explicitly exploits the co-existent and mutual exclusive relations among AUs from target labels or image features. They are referred to as model-based AU recognition methods. Tong et al.[6][7] used Gabor features and Adaboost to recognize each AU first, then they modeled the relations among AU labels by Dynamic Bayesian Network(DBN). Their method, however, learns the AU relationships from training data and such learned relations may not generalize well to a different database. To mitigate this problem, Li et al.[8] proposed to use a knowledge-driven model that satisfies specific constraints from AU relationships, then convert model parameter samples into pseudo-data and finally learned the pa-

rameters from the pseudo-data. Their method generalizes better across databases 
than the data-based models. Other than using DBN, Wang et al.[9] proposed a three-layer Restricted Boltzmann Machine (RBM) to capture global relations among all AUs, and to integrate the AU measurements with the high-level AU semantical relationships for $\mathrm{AU}$ recognition. More recently, Song et al.[10] modeled AU sparsity and co-occurrence using a Bayesian compressed sensing model. These work successfully model AU label relations, but ignore inherent AU relations in image features, which are crucial for $\mathrm{AU}$ analysis. Zhang et al.[11] utilized multi-task multiple kernel learning to detect multiple AUs in the same group simultaneously. Yuce et al.[12] applied the multi-label discriminant Laplacian embedding method for multiple AU recognition. These work successfully model AU relations from image features, or AU dependencies among AU labels. Therefore, current model-based AU recognition methods rarely exploit the dependencies inherent in both AU labels and image features to facilitate $\mathrm{AU}$ recognition [13][14].

Due to the difficulties of collecting data with AU intensity values and the limited available database, only a little research pays close attention to the intensity of facial actions. Moreover, most of them measure the intensity of each facial actions independently, such as $[15,16,17,18,19,20]$. In this paper, we refer to these methods as image-driven intensity estimation methods. Only recently, several works consider AU relations for AU intensity estimation. Li et al. [21] proposed using DBN to model AU relationships for measuring their intensities. Sandbach et al. [22] adopted Markov random field structures to model AU combination priors to estimate the intensity of AUs in the upper face region. Kaltwang et al. [23] proposed a generative latent tree model to estimate multiple AU intensity. They are referred to as model-based AU intensity estimation methods. Similar to AU recognition methods, few model-based AU intensity estimation methods exploits the dependencies inherent in both AU labels and image features.

To the best of our knowledge, this paper is the first work to recognize AUs and estimate AU intensity by exploring their relations at both feature and label levels [24]. By learning the shared features with MTL and modeling the dependencies among AU labels with BN, the proposed approach can exploit both top-down and bottom up relations among AUs to improve multiple AU classification and intensity estimation. 


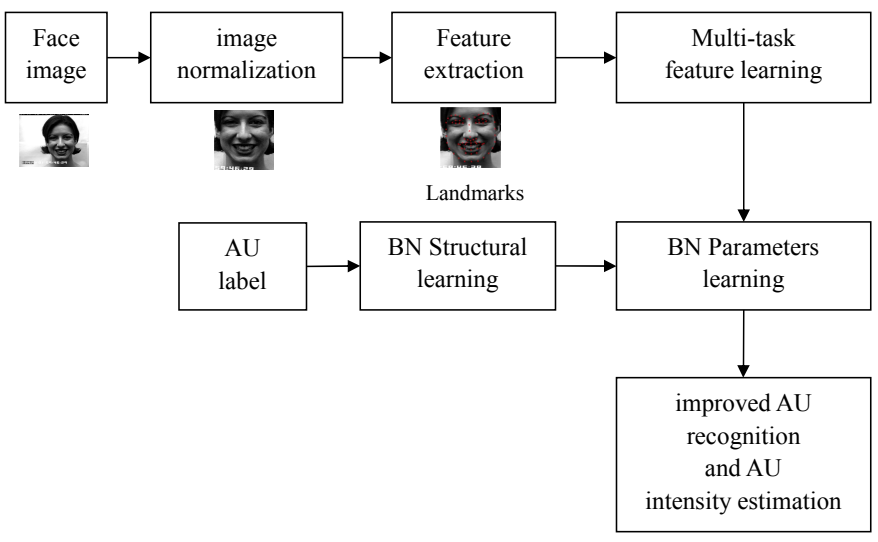

Figure 1: Framework of multiple AU analysis

\section{Multiple AU analysis approach}

Fig. 1 shows the framework of our multiple AU analysis approach. First, facial images are normalized and features are extracted. Second, multi-task feature learning is performed to recognize multiple AUs or estimate intensities of multiple AUs. Third, AU relations in labels are modeled by BN. Finally, we use the trained $\mathrm{BN}$ to refine the output of multi-task feature learning to improve AU classification or AU intensity estimation.

\subsection{Feature extraction}

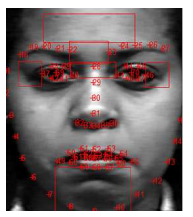

(a)

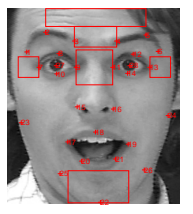

(b)

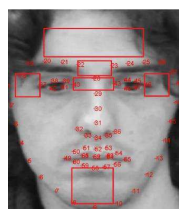

(c)

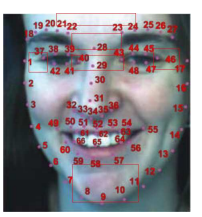

(d)

Figure 2: Landmark points and apperance feature blocks on CK+, MMI,DISFA and McMaster databases

Both geometric features and appearance features are extracted from the images. First, the face images are normalized to a fixed size of $330 \times 300$ according to the location of eyes. For the databases who provide feature points, we use these feature points as geometric features directly. For the databases without feature points, we detect the feature points using [25]. For appearance features, the 
Gabor features are extracted from the regions of the forehead, between the eyebrows, between the eyes, outer corner of eyes, and the lower jaw, as shown in Fig. 2. These appearance features present the transient features caused by the movement of muscles [26].

\subsection{Multiple AU recognition and intensity estimation by Multi-task learning}

Different from classical single-task learning, MTL trains multiple tasks jointly. Thus, one task in multi-task learning could benefit from learning other tasks.

Suppose there are $m$ AUs: $\Lambda=\left\{\lambda_{i}\right\}_{i=1}^{m}$, to be analyzed. We treat each of them as a single task. Let $L\left(T D_{i} ; \mathbf{w}_{i}\right)$ stand for the loss function of the $i$-th AU (i.e. $\left.\lambda_{i}\right)$ learning task on its training dataset $T D_{i}$, and $\mathbf{w}_{i}$ are the corresponding model parameters. For the AU intensity estimation task, we use the mean square errors as the loss function. For the AU classification task, we binarize the predicted value into AU states.

In standard learning paradigms, we analyze AUs independently and no information is shared among them, which is,

$$
\mathbf{W}^{\star}=\arg \min \sum_{i} L\left(T D_{i} ; \mathbf{w}_{i}\right)+\gamma\|\mathbf{W}\|_{F}^{2}
$$

where $\|\mathbf{W}\|_{F}^{2}$ is the squared Frobenius form: $\|\mathbf{W}\|_{F}^{2}=\sum_{i}\left\|\mathbf{w}_{i}\right\|_{2}^{2}$.

In MTL, we aim at finding a joint feature subspace $S$ where all AUs are well represented. Suppose the original feature $\mathbf{x}_{i}$ could be transferred to subspace $S$ by $\mathbf{s}_{i}=\mathbf{S}^{T} \mathbf{x}_{i}$, where $\mathbf{S} \in \mathbb{R}^{D \times D}$ is an orthogonal matrix. Thus, the decision function turns out to be three equivalent terms,

$$
\boldsymbol{\beta}_{i}^{T} \mathbf{S}_{i}=\boldsymbol{\beta}_{i}^{T} \mathbf{S}^{T} \mathbf{x}_{i}=\mathbf{w}_{i}^{T} \mathbf{x}_{i}
$$

So, $\mathbf{w}_{i}=\mathbf{S} \boldsymbol{\beta}_{i}$. S and $\boldsymbol{\beta}_{i}$ can be simultaneously solved by

$$
\mathbf{B}^{\star}, \mathbf{S}^{\star}=\arg \min \sum_{i} L\left(T D_{i} ; \boldsymbol{\beta}_{i}^{T} \mathbf{S}^{T}\right)+\gamma\|\mathbf{B}\|_{2,1}^{2}
$$

where $\|\mathbf{B}\|_{2,1}^{2}=\sum_{d=1}^{D} \sqrt{\sum_{i} \boldsymbol{\beta}_{d i}^{2}}$. This term calculates the 2-norm values of every dimension across all the AU recognition tasks, which captures the relationships in the feature-level space among AUs. Solving Eq. 3 can obtain the representation of the shared subspace $S$ simultaneously, which can achieve our goal. Besides, to reduce the complexity of the optimization algorithm, Argyriou et. al [27] proved 
that a closed solution could be achieved by transferring Eq. 3 into the following problem:

$$
\mathbf{W}^{\star}=\arg \min \sum_{i} L\left(T D_{i} ; \mathbf{w}_{i}\right)+\gamma\|\mathbf{W}\|_{\star}^{2},
$$

where $\|\mathbf{W}\|_{\star}$ is the trace norm of the parameter matrix.

To exploit the commonality among tasks, MTL is performed for a group of tasks, that have something in common. Suppose $m$ AU analysis can be divided into $P$ groups, and $q_{p i} \in\{0,1\}$ to indicate whether the $i$-th task is assigned to group $p$. Let $\mathbf{Q}$ be the group assignment matrix composed by $q_{p i}$ and $\mathbf{Q}_{p} \in \mathbb{R}^{n \times n}$ be a diagonal matrix with diagonal elements equal to $q_{p i} . \mathbf{W}_{p}$ is the parameter matrix for the $p$-th group, which is the parameter of the model. Multiple AU recognition task within a group are learned jointly, and the learning procedure for each group is independent. Kang et al. proposed an automatic grouping method to find the optimal Q in [28]. In our approach, before seeking the optimized model parameter $W$, we defined $Q$ manually according to the locations of AUs, as shown in Table 4. For each group, we get the optimized parameter $\mathbf{W}_{p}$ by solving the problem as follows:

$$
\mathbf{W}^{\star}=\arg \min \sum_{i} L\left(T D_{i} ; \mathbf{w}_{i}\right)+\gamma \sum_{p}\left\|\mathbf{W}_{p}\right\|_{\star}^{2}
$$

where $\left\|\mathbf{W}_{p}\right\|_{\star}^{2}=\operatorname{Trace}\left[\mathbf{W} \mathbf{Q}_{p}\left(\mathbf{W Q}_{p}\right)^{T}\right]^{\frac{1}{2}}$.

\subsection{AU Relationship Modeling from labels by $B N$}

As a probabilistic graphical model, BN can effectively capture the dependencies among variables in data. For this work, we use BN to capture the dependencies among AU labels.

\subsubsection{BN Structure and Parameters Learning}

A BN is a directed acyclic graph (DAG) $G=(\Lambda, E)$, where $\Lambda=\left\{\lambda_{i}\right\}_{i=1}^{m}$ represents a collection of $m$ nodes and $E$ denotes a collection of arcs.

Given the data of multiple AU labels $T D=\left\{\lambda_{i}^{j}\right\}$, where $i=1,2, \ldots, m$ is an index to the number of nodes, and $j=1,2, \ldots, n$ is index to the number samples. The structure and parameter learning is to find a structure $G$ that maximizes a score function. In this work, we employ the Bayesian Information Criterion (BIC) [29] score function which is defined as Eq. 6

$$
Q^{B I C}\left(G, \theta: G^{*}, \theta^{*}\right)=E_{G^{*}, \theta^{*}}[\log P(T D \mid G, \theta)]-\frac{\operatorname{Dim}(G)}{2} \log n
$$


where the first term is the log-likelihood function of structure $\mathrm{G}$ with respect to data $T D$, representing how well $G$ fits the data. The second term is a penalty relating to the complexity of the network, where $\operatorname{Dim}(G)$ is the number of independent parameters and $n$ is the number of samples. The BN structure learning algorithm proposed by Campos and Ji [30] are adopted to learn the dependencies among multiple AUs here.

For the AU classification task, after the BN structure is learned from the groundtruth labels, we link each node to the corresponding node for measurements as shown in Fig. 3 and Fig. 4. The parameters can be learned from the the groundtruth labels and their measurements of the training data.

For the AU intensity estimation task, a continuous BN consists of continuous variables should be learned from groundtruth AU intensities. However, the structure learning for a continuous $\mathrm{BN}$ is more complex than that for a discrete $\mathrm{BN}$. Therefore, in this paper, we propose a method to simplify the BN structure learning with continuous nodes. We first discretize each continuous AU intensities into the label with two classes based on the mean value of the ground-truth value. We use the discrete labels to learn the BN structure to model the relationships among AUs. After learned the BN structure based on binarized AUs, we link each node with two corresponding gaussian nodes representing the ground-truth label and measurement as shown in the red circle in Fig. 5 and Fig. 6.

During the training, the parameters of our BN network are estimated using the maximum-likelihood estimation (MLE), as shown in Eq. 7:

$$
\theta_{M L E}=\operatorname{argmax}_{\theta \in \Theta}\left(\frac{1}{n} \sum_{i=1}^{n} \sum_{j=1}^{m} \ln P\left(\lambda_{j}^{i} \mid p a\left(\lambda_{j}^{i}\right) ; \theta\right)\right)
$$

where $\theta$ is the parameter (i.e. the conditional probability of each node), $\lambda^{1}, \lambda^{2}, \ldots, \lambda^{n}$ are $\mathrm{n}$ independent samples, $\lambda_{j}^{i}$ is the value of the $j$ th node of the $i t h$ sample, $\operatorname{Pa}\left(\lambda_{j}^{i}\right)$ is the value of the $j t h$ node's parents of the $i t h$ sample.

\subsubsection{BN Inference}

A complete BN model is obtained after the parameter and structure learning. Given the AU measurements obtained from MTL, the true AU category or AU intensity of the input sample is estimated through BN inference. During the BN inference, the posterior probability can be estimated by combining the likelihood from measurement with the prior model.

For the AU classification, let $\lambda_{i}$ and $M_{i}, i \in\{1, \ldots, m\}$, denote the AU label variable and the corresponding measurement obtained from MTL respectively. 


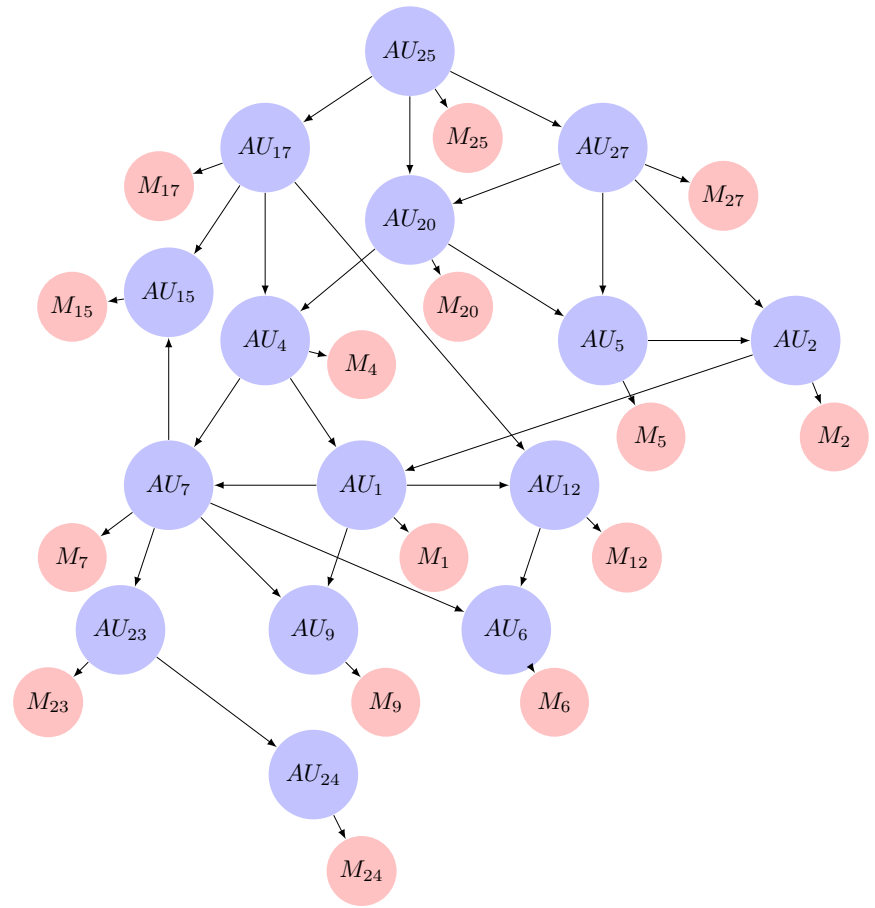

Figure 3: The BN model learned from the $\mathrm{CK}+$ database. "M" represents the $\mathrm{AU}$ measurement, "AU" represents the ground-truth AU label.

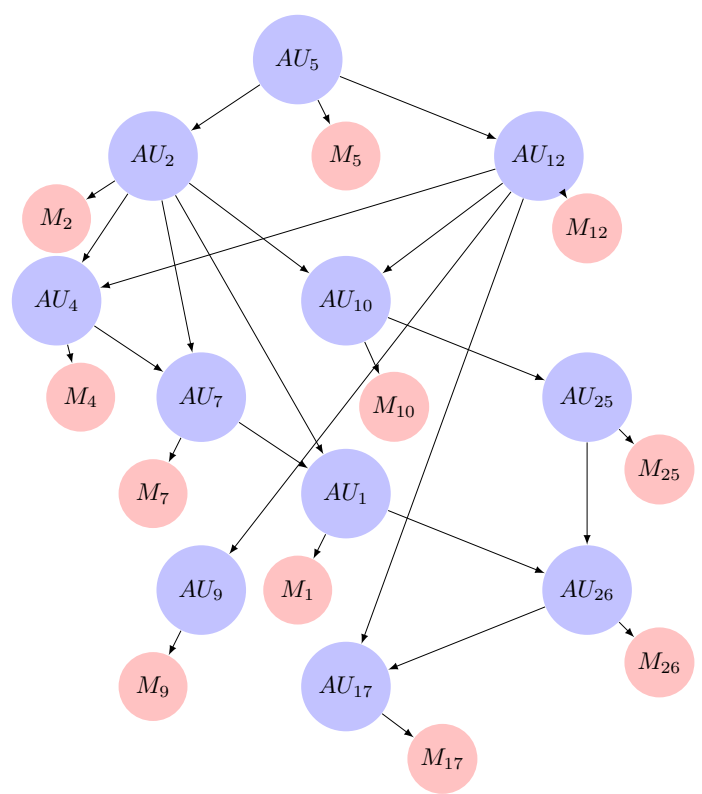

Figure 4: The BN model learned from the MMI database. "M" represents the AU measurement, "AU" represents the ground-truth AU label. 


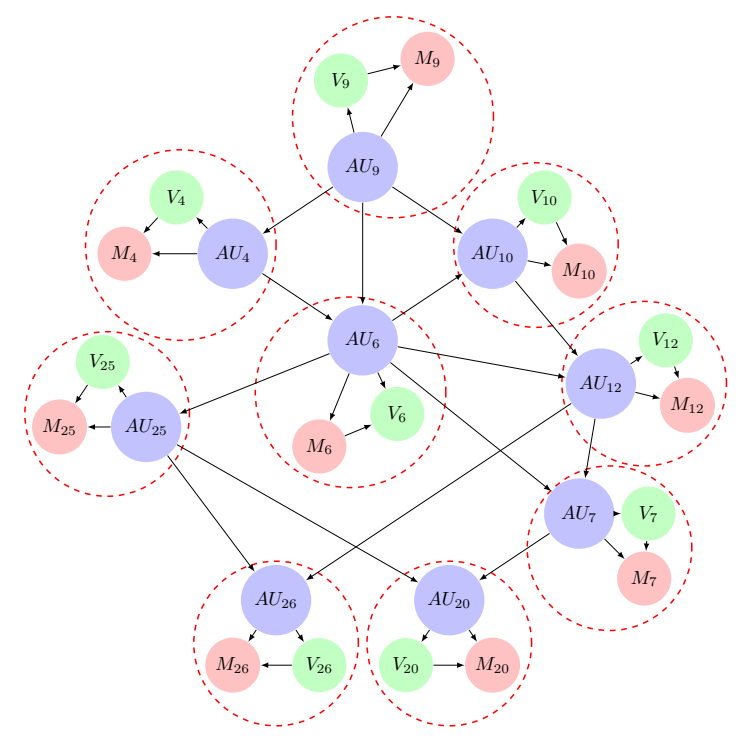

Figure 5: The BN model learned from the McMaster database. "M" represents the measurement of AU intensity, "AU" represents the ground-truth $\mathrm{AU}$ intensity. "V" represents the discrete value of AUs discretized based on the ground-truth intensity.

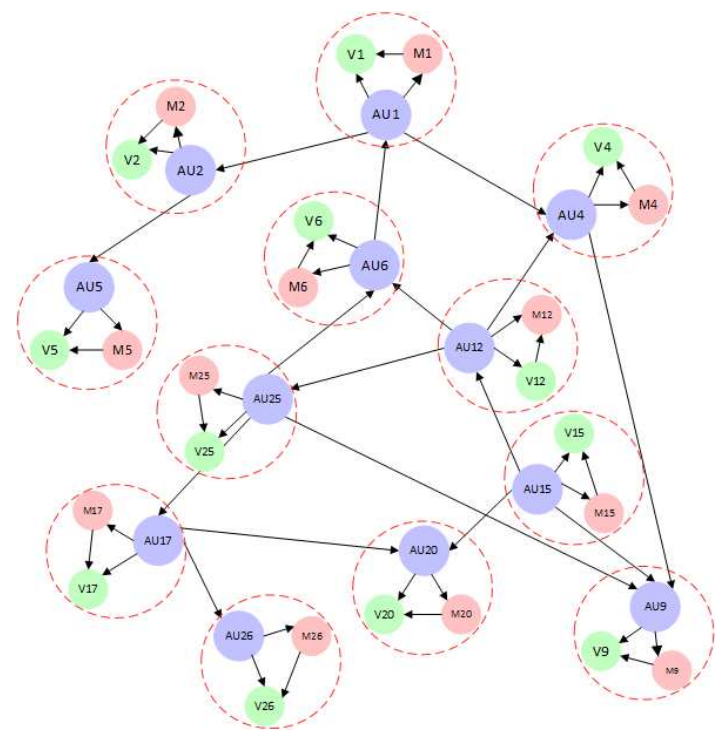

Figure 6: The BN model learned from the DISFA database. "M" represents the measurement of AU intensity, "AU" represents the ground-truth AU intensity. "V" represents the discrete value of AUs discretized based on the ground-truth intensity. 
Then, most probable explanation (MPE) [31] inference is used to estimate the joint probability of multiple AUs.

$$
\begin{aligned}
\mathbf{Y}^{\star} & =\underset{\lambda_{1}, \lambda_{2}, \ldots \lambda_{m}}{\arg \max } P\left(\lambda_{1}, \lambda_{2}, \ldots \lambda_{m} \mid M_{1}, \ldots, M_{m}\right) \\
& =\underset{\lambda_{1}, \lambda_{2}, \ldots \lambda_{m}}{\arg \max }\left(\prod_{i=1}^{m} P\left(M_{i} \mid \lambda_{i}\right) \prod_{i=1}^{m} P\left(\lambda_{i} \mid p a\left(\lambda_{i}\right)\right)\right)
\end{aligned}
$$

The first part of the equation is the likelihood of $\lambda_{j}$ given the measurements and the second part is the product of the conditional probabilities of each category node $\lambda_{j}$ given its parents $p a\left(\lambda_{j}\right)$, which are $\mathrm{BN}$ model parameters that have been learned. In AU classification, the inferred states of AUs are the states $\left(\mathbf{Y}^{\star}=\right.$ $\left.\left(\lambda_{1}, \ldots, \lambda_{m}\right)\right)$ with the highest probability given $M_{1}, \ldots, M_{m}$.

For AU intensity prediction, we use $L_{i}, \lambda_{i}$ and $M_{i}, i \in\{1,2, \ldots, m\}$, denote the discrete labels, the corresponding AU intensity values and the corresponding measurements gained from MTL respectively. Then,

$$
\begin{aligned}
\mathbf{Y}^{\star} & =\underset{L_{1}, \ldots, L_{m}, \lambda_{1}, \lambda_{2}, \ldots \lambda_{m}}{\arg \max } P\left(L_{1}, L_{2}, \ldots, L_{m}, \lambda_{1}, \lambda_{2}, \ldots, \lambda_{m} \mid M_{1}, M_{2}, \ldots, M_{m}\right) \\
& =\underset{L_{1}, \ldots, L_{m}, \lambda_{1}, \lambda_{2}, \ldots \lambda_{m}}{\arg \max }\left(\prod_{i=1}^{m} P\left(M_{i} \mid L_{i}, \lambda_{i}\right) \prod_{i=1}^{m} P\left(\lambda_{i} \mid L_{i}\right) \prod_{i=1}^{m} P\left(L_{i} \mid p a\left(L_{i}\right)\right)\right.
\end{aligned}
$$

The condition probability in the equation is learned in the training phase. The inferred results are $\mathbf{Y}^{\star}=\left(\lambda_{1}, \ldots, \lambda_{m}, L_{1}, \ldots, L_{m}\right)$ with the highest probability given $M_{1}, \cdots, M_{m}$. In practice, we use the junction tree algorithm [32] to estimate the posterior probability effectively.

\section{Experiments and results}

\subsection{Experimental conditions}

To validate our approach, we conduct $\mathrm{AU}$ recognition experiments on the $\mathrm{CK}+$ database [33] and the MMI database [34], and AU intensity estimation on the McMaster database [35] and the DISFA database [36].

The $\mathrm{CK}+$ database consists of 593 posed expression image sequences, starting from the neutral frame and ending at the peak frame, from 123 subjects. 593 apex images signed with AU labels are selected, and the AUs, whose positive sample number is larger than 50, are used in the experiment, which are: AU1, AU2, AU4, AU5, AU6, AU7, AU9, AU12, AU15, AU17, AU20, AU23, AU24, AU25, 
and AU27. The sample distribution over AU states on the $\mathrm{CK}+$ database is shown in Table 1. A 10-fold cross validation is adopted.

The MMI database consists of over 2900 videos and high-resolution images of 75 subjects. It contains recordings of the full temporal pattern of facial expressions, from neutral, through a series of onset, apex, and offset phases, and finally back again to a neutral face. In this experiment, 623 samples are selected, and 11 AUs are used in the experiment : AU1, AU2, AU4, AU5, AU7, AU9, AU10, AU12, AU17, AU25, and AU26. The sample distribution over AU states on the MMI database is shown in Table 1. A 10-fold cross validation is adopted.

The McMaster database contains face videos of patients suffering from shoulder pain. Totally, 200 sequences of 25 subjects are recorded (48,398 frames). AU intensities are provided for each frame. AUs 4,6,7,9,10,12,20,25,26 and 27 are labeled on 6 levels (0-5) and AU 43 are labeled 2 levels(present or not). The sample distribution over AU intensity levels are listed in Table 2. For AU 27, its data are very unbalanced and AU 43 are labeled on only 2 levels, therefore we use the rest 9 AUs for our experiment. We adopt the leave-one-subject cross validation for our AU intensity estimation experiment.

The DISFA database [36] contains spontaneous facial expressions of 27 young adults while watching emotion eliciting videos. Each facial image has been annotated with six scale AU intensity for 12 AUs (i.e. AU1, AU2, AU4, AU5, AU6, AU9, AU12, AU15, AU17, AU20, AU25, and AU26) by an expert FACS rater.The samples distribution of database is shown in Table 3. In our experiment, the 19850 samples whose sum of intensity is larger than 10 are selected. The leave-one-subject cross validation is adopted.

We divided the AUs into several groups according to their locations, as shown in Table 4. For the AUs in the same group, we learn the shared features using the MTL described in Section 3.2.

To demonstrate the effectiveness of our methods, three experiments are conducted: AU recognition/AU intensity prediction considering each AU individually (single task), AU recognition/AU intensity prediction using MTL (MTL), and our approach consider AU relations from both features and labels (MTL+BN).

For AU classification, we calculate metrics from two aspects: example-based view and label-based view. For example-based view, we calculate accuracy and F1-score on each AU as well as the average of them. For label-based measures, we adopt macro F1-score and micro F1-score [37] . For AU intensity prediction, we use three metrics: the Pearson correlation coefficient, the intraclass correlation coefficient (ICC)[38] and the mean squared error(MSE). 
Table 1: Sample distribution over AU states on the CK+ and MMI databases

\begin{tabular}{|c|c|c|c|c|}
\hline & \multicolumn{2}{|c|}{ CK+ database } & \multicolumn{2}{c|}{ MMI database } \\
\hline Label & 0 & 1 & 0 & 1 \\
\hline AU1 & 418 & 175 & 428 & 195 \\
\hline AU2 & 476 & 117 & 393 & 230 \\
\hline AU4 & 399 & 194 & 398 & 225 \\
\hline AU5 & 491 & 102 & 388 & 235 \\
\hline AU6 & 470 & 123 & - & - \\
\hline AU7 & 472 & 121 & 448 & 175 \\
\hline AU9 & 518 & 75 & 518 & 105 \\
\hline AU10 & - & - & 523 & 100 \\
\hline AU12 & 462 & 131 & 503 & 120 \\
\hline AU15 & 498 & 95 & - & - \\
\hline AU17 & 390 & 203 & 478 & 145 \\
\hline AU20 & 514 & 79 & - & - \\
\hline AU23 & 533 & 60 & - & - \\
\hline AU24 & 535 & 58 & - & - \\
\hline AU25 & 269 & 324 & 173 & 450 \\
\hline AU26 & - & - & 328 & 295 \\
\hline AU27 & 512 & 81 & - & - \\
\hline
\end{tabular}

Table 2: Sample distribution over AU intensity levels on the McMaster Database

\begin{tabular}{|c|c|c|c|c|c|c|}
\hline Intensity & 0 & 1 & 2 & 3 & 4 & 5 \\
\hline AU4 & 47324 & 202 & 509 & 225 & 74 & 64 \\
\hline AU6 & 42841 & 1776 & 1663 & 1327 & 681 & 110 \\
\hline AU7 & 45034 & 1360 & 991 & 608 & 305 & 100 \\
\hline AU9 & 47975 & 93 & 151 & 68 & 76 & 35 \\
\hline AU10 & 47873 & 171 & 208 & 63 & 61 & 22 \\
\hline AU12 & 41511 & 2145 & 1799 & 2158 & 736 & 49 \\
\hline AU20 & 47692 & 286 & 282 & 118 & 0 & 20 \\
\hline AU25 & 45992 & 766 & 803 & 611 & 138 & 88 \\
\hline AU26 & 46306 & 430 & 918 & 265 & 478 & 1 \\
\hline AU27 & 48380 & 6 & 3 & 3 & 6 & 0 \\
\hline AU43 & 45964 & 2434 & - & - & - & - \\
\hline
\end{tabular}


Table 3: Sample distribution over AU intensity levels on the DISFA Database

\begin{tabular}{|c|c|c|c|c|c|c|}
\hline Intensity & 0 & 1 & 2 & 3 & 4 & 5 \\
\hline AU1 & 14942 & 344 & 286 & 2432 & 1291 & 555 \\
\hline AU2 & 16388 & 144 & 83 & 2124 & 752 & 359 \\
\hline AU4 & 12518 & 478 & 744 & 2335 & 2768 & 1007 \\
\hline AU5 & 19168 & 213 & 192 & 189 & 62 & 26 \\
\hline AU6 & 9738 & 2734 & 3281 & 3367 & 589 & 141 \\
\hline AU9 & 16120 & 377 & 772 & 2277 & 283 & 21 \\
\hline AU12 & 9312 & 816 & 796 & 6301 & 2453 & 172 \\
\hline AU15 & 16815 & 1825 & 385 & 780 & 45 & 0 \\
\hline AU17 & 16281 & 1160 & 758 & 1540 & 100 & 11 \\
\hline AU20 & 18788 & 58 & 323 & 681 & 0 & 0 \\
\hline AU25 & 4827 & 860 & 1120 & 7720 & 4464 & 859 \\
\hline AU26 & 11493 & 3923 & 1701 & 2327 & 234 & 172 \\
\hline
\end{tabular}

Table 4: AU group definitions

\begin{tabular}{|c|c|c|c|c|}
\hline $\begin{array}{c}\text { Groups } \\
\text { (Facial Regions) }\end{array}$ & CK+ & MMI & McMaster & DISFA \\
\hline \multirow{3}{*}{ Eye } & $\begin{array}{c}\text { AU1, AU2, } \\
\text { AU4, AU5, } \\
\text { AU7 }\end{array}$ & $\begin{array}{c}\text { AU1, AU2, } \\
\text { AU4, AU5, } \\
\text { AU7 }\end{array}$ & AU4, AU7 & $\begin{array}{c}\text { AU1, AU2, } \\
\text { AU4, AU5 }\end{array}$ \\
\hline \multirow{5}{*}{ Mouth and chin } & $\begin{array}{c}\text { AU12, AU15, } \\
\text { AU17, AU20, }\end{array}$ & $\begin{array}{c}\text { AU10, AU12, } \\
\text { AU17, AU25, } \\
\text { AU23, AU24, } \\
\text { AU25, AU27 }\end{array}$ & $\begin{array}{c}\text { AU26,AU12, } \\
\text { AU20,AU25, } \\
\text { AU26 }\end{array}$ & $\begin{array}{c}\text { AU12, AU15, } \\
\text { AU17, AU20, } \\
\text { AU25, AU26 }\end{array}$ \\
\hline Cheek and nose & AU6, AU9 & AU9 & AU6,AU9 & AU6,AU9 \\
\hline
\end{tabular}


Table 5: AU recognition on $\mathrm{CK}+$ database from example-based view

\begin{tabular}{|c|c|c|c|c|c|}
\hline $\mathrm{AU}$ & $\begin{array}{c}\text { Accuracy (\%) } \\
\text { /F1-score of } \\
\text { single task }\end{array}$ & $\begin{array}{c}\text { Accuracy (\%) } \\
\text { /F1-score of } \\
\text { single task + BN }\end{array}$ & $\begin{array}{c}\text { Accuracy }(\%) \\
\text { /F1-score } \\
\text { of MTL }\end{array}$ & $\begin{array}{c}\text { Accuracy }(\%) \\
\text { /F1-score of } \\
\text { MTL + BN }\end{array}$ & $\begin{array}{c}\text { Accu. } \\
(\%) \text { in } \\
{[4]}\end{array}$ \\
\hline \hline 1 & $84.82 / 0.6875$ & $84.49 / 0.6783$ & $\mathbf{8 7 . 1 8 / 0 . 7 6 1 0}$ & $84.65 / 0.6873$ & 97.5 \\
2 & $92.75 / 0.7795$ & $92.75 / 0.7795$ & $\mathbf{9 3 . 7 6 / 0 . 8 2 1 3}$ & $92.75 / 0.7882$ & 87.1 \\
4 & $80.27 / 0.6139$ & $79.60 / 0.6033$ & $73.86 / \mathbf{0 . 6 3 0 1}$ & $\mathbf{7 8 . 5 8 / 0 . 6 3 1 9}$ & 97.4 \\
5 & $89.88 / 0.6341$ & $\mathbf{9 0 . 7 3 / 0 . 6 7 8 4}$ & $\mathbf{9 0 . 7 3 / 0 . 6 8 9 3}$ & $\mathbf{9 1 . 4 0 / 0 . 7 2 1 3}$ & 87.0 \\
6 & $87.18 / 0.6162$ & $87.18 / 0.6162$ & $\mathbf{8 8 . 5 3 / 0 . 7 1 6 7}$ & $\mathbf{8 8 . 8 7 / 0 . 7 1 0 5}$ & 80.2 \\
7 & $83.98 / 0.3949$ & $83.98 / \mathbf{0 . 4 1 7 2}$ & $82.80 / \mathbf{0 . 5 5 6 5}$ & $\mathbf{8 4 . 3 2} / 0.5550$ & 89.1 \\
9 & $89.71 / 0.3297$ & $89.71 / 0.3297$ & $\mathbf{9 7 . 3 0 / 0 . 8 8 8 9}$ & $96.96 / 0.8732$ & 100.0 \\
12 & $90.22 / 0.7456$ & $90.22 / \mathbf{0 . 7 4 7 8}$ & $90.22 / \mathbf{0 . 7 8 5 2}$ & $\mathbf{9 3 . 4 2 / 0 . 8 4 8 2}$ & 92.4 \\
15 & $84.15 / 0.0208$ & $\mathbf{8 0 . 7 8 / 0 . 5 5 1 2}$ & $\mathbf{9 1 . 0 6 / 0 . 6 9 0 1}$ & $\mathbf{9 1 . 7 4 / 0 . 7 0 3 0}$ & 91.0 \\
17 & $87.86 / 0.8182$ & $87.18 / 0.8100$ & $85.67 / 0.7826$ & $\mathbf{8 9 . 5 4 / 0 . 8 4 8 0}$ & 89.0 \\
20 & $87.18 / 0.0952$ & $\mathbf{8 8 . 0 3 / 0 . 2 0 2 2}$ & $\mathbf{9 0 . 0 5 / 0 . 5 6 9 3}$ & $\mathbf{9 2 . 7 5 / 0 . 6 7 1 8}$ & 91.1 \\
23 & $89.88 / 0.0000$ & $89.88 / 0.0000$ & $\mathbf{9 1 . 7 4 / 0 . 4 3 6 8}$ & $\mathbf{9 1 . 9 1 / 0 . 4 2 8 6}$ & 81.3 \\
24 & $90.22 / 0.0000$ & $90.22 / 0.0000$ & $90.05 / \mathbf{0 . 2 1 3 3}$ & $\mathbf{9 0 . 8 9 / 0 . 3 2 5 0}$ & N/A \\
25 & $88.53 / 0.8828$ & $88.53 / 0.8828$ & $82.97 / 0.8308$ & $\mathbf{9 2 . 0 7 / 0 . 9 1 6 2}$ & 90.7 \\
27 & $96.12 / 0.8456$ & $95.95 / 0.8400$ & $\mathbf{9 7 . 1 3 / 0 . 9 3 1 4}$ & $\mathbf{9 7 . 3 0 / 0 . 9 0 0 0}$ & N/A \\
\hline \hline Ave & $88.18 / 0.4976$ & $87.95 / \mathbf{0 . 5 4 2 4}$ & $\mathbf{9 0 . 3 3 / 0 . 6 7 8 9}$ & $\mathbf{9 0 . 4 8 / 0 . 7 0 7 2}$ & 90.29 \\
\hline
\end{tabular}

\subsection{Experimental results and analyses for AU classification}

\subsubsection{Analyses from Example-based view}

Table 5 provides the AU recognition results on the $\mathrm{CK}+$ database from examplebased view. From Table 5, we can find the follows:

First, comparing the AU recognition as single task and AU recognition as multi-task, the accuracies of 9 AUs and the F1-scores of 13 AUs increase under the help of shared feature learning, demonstrating the effectiveness of our proposed multi-task AU recognition with the facial-region grouping strategy. Specifically, there exist a significant improvement for AU9, whose F1-score increases by 0.5592 , and accuracy increases by $7.59 \%$. Furthermore, the performance of its group partener - AU6 is also improved, with an increase of 0.1005 in F1score and $1.53 \%$ in accuracy. Such improvement indicates that each AU in this group benefits from the shared feature space, especially for the AUs that take a small fraction of the samples, such as AU9, whose proportion of the total sample is $12.64 \%$. Similar phenomena can be found in AU15(16.02\%), AU20(13.32\%), AU23 (10.11\%) and AU24(9.78\%), whose F1-scores increase by 0.6693, 0.4741, 0.4368 and 0.2133 respectively. In addition, the average accuracies and F1-scores increase by $2.15 \%$ and 0.1813 , further confirming the effectiveness of MTL.

Second, comparing the result of our method using both MTL and BN with the one using MTL, there are 12 AUs with improvement in accuracy and 8 AUs with increase in F1-scores. The most significant improvement occurs in the F1-score of AU24, whose increase is 0.1117 . From Fig. 3, we can find that the only link to 
Table 6: AU dependencies on $\mathrm{CK}+$ database

\begin{tabular}{|l|l|l|l|l|l|l|l|l|l|l|l|l|l|l|l|l|}
\hline$\lambda_{i} \lambda_{j}$ & AU1 & AU2 & AU4 & AU5 & AU6 & AU7 & AU9 & AU12 & AU15 & AU17 & AU20 & AU23 & AU24 & AU25 & AU27 \\
\hline AU1 & 1.00 & 1.00 & 0.35 & 0.85 & 0.07 & 0.14 & 0.00 & 0.07 & 0.39 & 0.24 & 0.57 & 0.13 & 0.05 & 0.39 & 0.89 \\
\hline AU2 & 0.67 & 1.00 & 0.10 & 0.79 & 0.01 & 0.01 & 0.00 & 0.04 & 0.14 & 0.08 & 0.24 & 0.05 & 0.05 & 0.31 & 0.89 \\
\hline AU4 & 0.39 & 0.16 & 1.00 & 0.25 & 0.28 & 0.81 & 0.67 & 0.07 & 0.40 & 0.63 & 0.66 & 0.75 & 0.62 & 0.18 & 0.02 \\
\hline AU5 & 0.50 & 0.69 & 0.13 & 1.00 & 0.02 & 0.05 & 0.01 & 0.04 & 0.03 & 0.05 & 0.28 & 0.12 & 0.00 & 0.29 & 0.75 \\
\hline AU6 & 0.05 & 0.01 & 0.18 & 0.03 & 1.00 & 0.38 & 0.32 & 0.63 & 0.01 & 0.11 & 0.20 & 0.15 & 0.09 & 0.26 & 0.00 \\
\hline AU7 & 0.10 & 0.01 & 0.51 & 0.06 & 0.37 & 1.00 & 0.64 & 0.08 & 0.07 & 0.35 & 0.34 & 0.58 & 0.55 & 0.12 & 0.00 \\
\hline AU9 & 0.00 & 0.00 & 0.26 & 0.01 & 0.20 & 0.40 & 1.00 & 0.03 & 0.04 & 0.26 & 0.03 & 0.17 & 0.21 & 0.04 & 0.00 \\
\hline AU12 & 0.05 & 0.04 & 0.05 & 0.05 & 0.67 & 0.09 & 0.05 & 1.00 & 0.00 & 0.01 & 0.13 & 0.00 & 0.03 & 0.29 & 0.04 \\
\hline AU15 & 0.21 & 0.11 & 0.20 & 0.03 & 0.01 & 0.06 & 0.05 & 0.00 & 1.00 & 0.45 & 0.01 & 0.15 & 0.16 & 0.01 & 0.01 \\
\hline AU17 & 0.28 & 0.15 & 0.65 & 0.11 & 0.19 & 0.60 & 0.69 & 0.02 & 0.97 & 1.00 & 0.08 & 0.80 & 0.78 & 0.03 & 0.00 \\
\hline AU20 & 0.26 & 0.16 & 0.27 & 0.22 & 0.13 & 0.22 & 0.03 & 0.08 & 0.01 & 0.03 & 1.00 & 0.02 & 0.00 & 0.23 & 0.01 \\
\hline AU23 & 0.05 & 0.03 & 0.23 & 0.07 & 0.07 & 0.29 & 0.13 & 0.00 & 0.09 & 0.24 & 0.01 & 1.00 & 0.59 & 0.00 & 0.01 \\
\hline AU24 & 0.02 & 0.03 & 0.19 & 0.00 & 0.04 & 0.26 & 0.16 & 0.02 & 0.09 & 0.22 & 0.00 & 0.57 & 1.00 & 0.00 & 0.00 \\
\hline AU25 & 0.71 & 0.86 & 0.30 & 0.91 & 0.69 & 0.32 & 0.17 & 0.71 & 0.02 & 0.04 & 0.96 & 0.02 & 0.00 & 1.00 & 1.00 \\
\hline AU27 & 0.41 & 0.62 & 0.01 & 0.60 & 0.00 & 0.00 & 0.00 & 0.02 & 0.01 & 0.00 & 0.01 & 0.02 & 0.00 & 0.25 & 1.00 \\
\hline
\end{tabular}

AU24 is AU23. Thus, the improvement may be cause by the dependency between them. Furthermore, the average accuracies and F1-scores increase by $0.15 \%$ and 0.0283 , demonstrating that the BN model can improve the results from MTL.

Fig. 3 shows the learned BN structure, and Table 6 lists the dependencies between each AU pair: $P\left(\lambda_{j} \mid \lambda_{i}\right)$, measuring the probability of label $\lambda_{j}$ happens, given label $\lambda_{i}$ happens. Comparing the learned BN with the dependency table, we find that the label pairs whose conditional probabilities are top ranked or bottom ranked are linked in the $\mathrm{BN}$ in most cases. For example, the link from AU1 to AU9 shows the mutual exclusive relationship, since $P(A U 9 \mid A U 1)$ is zero. While the link from AU2 to AU1 represents the strong co-occurrence relationship between AU1 and AU2, since $P(A U 1 \mid A U 2)$ is 1.0. Therefore, the learned BN can systematically capture the relations among AU labels. Thus, the learned BN can calibrate the AU recognition results from MTL.

Third, our proposed AU recognition method considering AU relations in both feature and label space performs best among the three AU recognition experiments, with the highest average accuracy and F1-scores.

Table 7 provides the AU recognition results on the MMI database from examplebased view. Similar to the analysis on the $\mathrm{CK}+$ database, we can find the following from Table 7:

First, comparing the AU recognition as single task and AU recognition as multi-task, the accuracies and F1-scores of 9 AUs increase by sharing feature spaces, which shows the effectiveness of our proposed multi-task AU recognition with the facial-region grouping strategy. Specifically, there exist a significant improvement for AU1, whose accuracy increases by $17.49 \%$ and $\mathrm{F} 1$-score increases by 0.3485 . Furthermore, the performance of its group partner - AU7 are also improved, with a increase of 0.0398 in F1-score and $1.60 \%$ in accuracy. In addition, the average accuracies and F1-scores increase by $1.86 \%$ and 0.0411 , which 
Table 7: AU recognition on MMI database from example-based view

\begin{tabular}{|c|c|c|c|c|}
\hline $\mathrm{AU}$ & $\begin{array}{c}\text { Accuracy (\%) } \\
\text { /F1-score of } \\
\text { single task }\end{array}$ & $\begin{array}{c}\text { Accuracy (\%) } \\
\text { /F1-score } \\
\text { of MTL }\end{array}$ & $\begin{array}{c}\text { Accuracy (\%) } \\
\text { /F1-score of } \\
\text { MTL + BN }\end{array}$ & $\begin{array}{c}\text { F1- } \\
\text { score } \\
\text { in [39 }\end{array}$ \\
\hline \hline 1 & $80.42 / 0.6188$ & $\mathbf{9 7 . 9 1 / 0 . 9 6 7 3}$ & $\mathbf{9 8 . 5 6 / 0 . 9 7 7 1}$ & 0.850 \\
\hline 2 & $96.15 / 0.9462$ & $\mathbf{9 6 . 7 9 / 0 . 9 5 5 2}$ & $96.79 / 0.9552$ & 0.822 \\
\hline 4 & $97.27 / 0.9623$ & $\mathbf{9 7 . 5 9 / 0 . 9 6 6 7}$ & $96.63 / 0.9538$ & 0.828 \\
\hline 5 & $95.18 / 0.9336$ & $94.22 / 0.9204$ & $94.22 / 0.9204$ & 0.825 \\
\hline 7 & $92.46 / 0.8498$ & $\mathbf{9 4 . 0 6 / 0 . 8 8 9 6}$ & $\mathbf{9 4 . 2 2 / 0 . 8 9 2 9}$ & 0.810 \\
\hline 9 & $96.47 / 0.8842$ & $\mathbf{9 6 . 6 3 / 0 . 8 8 8 9}$ & $96.63 / 0.8889$ & 0.959 \\
\hline 10 & $95.35 / 0.8398$ & $\mathbf{9 6 . 1 5 / 0 . 8 7 7 6}$ & $\mathbf{9 6 . 3 1 / 0 . 8 8 0 8}$ & 0.877 \\
\hline 12 & $98.56 / 0.9610$ & $97.91 / 0.9482$ & $97.91 / 0.9469$ & 0.958 \\
\hline 17 & $95.35 / 0.8897$ & $\mathbf{9 5 . 8 3 / 0 . 9 0 9 7}$ & $95.83 / 0.9097$ & 0.828 \\
\hline 25 & $92.78 / 0.8673$ & $\mathbf{9 3 . 1 0 / 0 . 8 7 0 1}$ & $92.78 / 0.8640$ & 0.795 \\
\hline 26 & $88.12 / 0.8737$ & $\mathbf{8 8 . 2 8 / 0 . 8 8 4 7}$ & $88.12 / 0.8814$ & 0.885 \\
\hline \hline Ave & $93.46 / 0.8751$ & $\mathbf{9 5 . 3 2 / 0 . 9 1 6 2}$ & $95.27 / 0.9156$ & 0.858 \\
\hline
\end{tabular}

further confirming the effectiveness of MTL.

Second, comparing the result of our method with the one using MTL, there are 3 AUs with improvement in accuracy and F1-score, and all of the improvement are less than 1\%/0.01. Furthermore, the average accuracies and F1-score of our proposed method slightly decrease by $0.05 \%$ and 0.0006 , which indicates a minor improvement with the BN structure. However, the accuracy and F1-score of AU1 increase in both single task and MTL results, which shows that the structure learned from MMI could improve the recognition performance on AU1.

Fig. 4 shows the learned BN structure, and Table 8 lists all the dependencies. Comparing the learned $\mathrm{BN}$ with the dependency table, we find that the label pairs whose conditional probabilities are top ranked or bottom ranked are linked in the BN in most cases. For example, the link from AU5 to AU12 shows the mutual exclusive relationship, since $P(A U 12 \mid A U 5)$ is 0.04 . While the link from AU25 to AU26 represents the strong co-occurrence relationship between AU25 and AU26, since $P(A U 25 \mid A U 26)$ is 1.0. Therefore, the learned BN can systematically capture the relations among AU labels. Thus, the learned BN can improve the recognition results from both single task and MTL, especially for AU1.

From Table 5 and Table 7, we can find that multi-task AU recognition is better than single task AU recognition for both databases; the learned BN structures improve the recognition performance of single task for both databases, but it only help multi-task AU recognition on the $\mathrm{CK}+$ database, not the MMI database. It may indicate that the learned relations among AUs can help AU recognition especially when the measurements are poor. 
Table 8: AU dependencies on MMI database

\begin{tabular}{|c|c|c|c|c|c|c|c|c|c|c|c|}
\hline$\lambda_{i} \lambda_{j}$ & AU1 & AU2 & AU4 & AU5 & AU7 & AU9 & AU10 & AU12 & AU17 & AU25 & AU26 \\
\hline AU1 & 1.00 & 0.87 & 0.23 & 0.67 & 0.18 & 0.00 & 0.05 & 0.10 & 0.15 & 0.77 & 0.62 \\
\hline AU2 & 0.74 & 1.00 & 0.13 & 0.80 & 0.07 & 0.02 & 0.02 & 0.09 & 0.15 & 0.85 & 0.65 \\
\hline AU4 & 0.20 & 0.13 & 1.00 & 0.24 & 0.60 & 0.20 & 0.27 & 0.00 & 0.40 & 0.47 & 0.24 \\
\hline AU5 & 0.55 & 0.79 & 0.23 & 1.00 & 0.17 & 0.13 & 0.11 & 0.04 & 0.13 & 0.83 & 0.60 \\
\hline AU7 & 0.20 & 0.09 & 0.77 & 0.23 & 1.00 & 0.23 & 0.23 & 0.00 & 0.34 & 0.49 & 0.34 \\
\hline AU9 & 0.00 & 0.05 & 0.43 & 0.29 & 0.38 & 1.00 & 0.43 & 0.00 & 0.38 & 0.76 & 0.52 \\
\hline AU10 & 0.10 & 0.05 & 0.60 & 0.25 & 0.40 & 0.45 & 1.00 & 0.05 & 0.30 & 0.85 & 0.45 \\
\hline AU12 & 0.17 & 0.17 & 0.00 & 0.08 & 0.00 & 0.00 & 0.04 & 1.00 & 0.00 & 0.83 & 0.46 \\
\hline AU17 & 0.21 & 0.24 & 0.62 & 0.21 & 0.41 & 0.28 & 0.21 & 0.00 & 1.00 & 0.45 & 0.21 \\
\hline AU25 & 0.33 & 0.43 & 0.23 & 0.43 & 0.19 & 0.18 & 0.19 & 0.22 & 0.14 & 1.00 & 0.66 \\
\hline AU26 & 0.41 & 0.51 & 0.19 & 0.47 & 0.20 & 0.19 & 0.15 & 0.19 & 0.10 & 1.00 & 1.00 \\
\hline
\end{tabular}

Table 9: Label-based AU recognition results on $\mathrm{CK}+$ and $\mathrm{MMI}$ databases

\begin{tabular}{|c|c|c|c|c|}
\hline Database & $\begin{array}{c}\text { Evaluation } \\
\text { Metric }\end{array}$ & $\begin{array}{c}\text { Single } \\
\text { Task }\end{array}$ & MTL & MTL+BN \\
\hline \hline \multirow{2}{*}{ CK+ } & Macro F1 & 0.8788 & 0.9071 & 0.9148 \\
\cline { 2 - 5 } & Micro F1 & 0.8805 & 0.9133 & 0.9172 \\
\hline \multirow{2}{*}{ MMI } & Macro F1 & 0.9279 & 0.9612 & 0.9584 \\
\cline { 2 - 5 } & Micro F1 & 0.9313 & 0.9652 & 0.9632 \\
\hline
\end{tabular}

\subsubsection{Analyses from Label-based view}

Table 9 shows the label-based results on the $\mathrm{CK}+$ and the MMI databases. It is clear that all the F1 measures of MTL is higher than those of single task, which indicates that MTL outperforms the classic method. Besides, the F1-measures of our method on $\mathrm{CK}+$ is the highest among all the methods, which also demonstrates the effectiveness of our method. Specifically, The macro/micro F1-score of MTL+BN on MMI database is only 0.0028/0.0020 lower comparing with MTL, which could be treated as an equivalent result.

\subsubsection{Cross database experiments}

To further validate the generalization ability of our proposed approach, we perform cross-database experiments. Since the feature points provided by the $\mathrm{CK}+$ database and MMI database are different, we can not validate the generalization ability of multi-task learning due to different features for different databases. Therefore, we only verify the generalization ability of the learned BN structures.

Table 10 and 11 shows the cross-database experiment results on $\mathrm{CK}+$ and MMI database. It is clear that for single task, the average F1-score increases when 
Table 10: Cross database AU recognition on the structure learnt from $\mathrm{CK}+$ database

\begin{tabular}{|l|c|c|c|c|}
\hline $\begin{array}{c}\text { AU in } \\
\text { MMI }\end{array}$ & $\begin{array}{c}\text { Accuracy (\%) } \\
\text { /F1-score of } \\
\text { single task }\end{array}$ & $\begin{array}{c}\text { Accuracy (\%) } \\
\text { /F1-score of } \\
\text { single task + BN }\end{array}$ & $\begin{array}{c}\text { Accuracy (\%) } \\
\text { /F1-score } \\
\text { of MTL }\end{array}$ & $\begin{array}{c}\text { Accuracy (\%) } \\
\text { /F1-score of } \\
\text { MTL + BN }\end{array}$ \\
\hline 1 & $80.42 / 0.6188$ & $\mathbf{8 8 . 9 2 / 0 . 8 3 1 3}$ & $97.91 / 0.9673$ & $97.91 / 0.9673$ \\
\hline 2 & $96.15 / 0.9462$ & $96.15 / 0.9462$ & $96.79 / 0.9552$ & $96.15 / 0.9467$ \\
\hline 4 & $97.27 / 0.9623$ & $97.27 / 0.9623$ & $97.59 / 0.9667$ & $97.59 / 0.9667$ \\
\hline 5 & $95.18 / 0.9336$ & $95.18 / 0.9336$ & $94.22 / 0.9204$ & $94.22 / 0.9204$ \\
\hline 7 & $92.46 / 0.8498$ & $91.97 / 0.8418$ & $94.06 / 0.8896$ & $94.06 / 0.8896$ \\
\hline 9 & $96.47 / 0.8842$ & $96.47 / 0.8842$ & $96.63 / 0.8889$ & $96.63 / 0.8889$ \\
\hline 12 & $98.56 / 0.9610$ & $98.56 / 0.9610$ & $97.91 / 0.9482$ & $97.91 / 0.9482$ \\
\hline 17 & $95.35 / 0.8897$ & $95.35 / 0.8897$ & $95.83 / 0.9097$ & $95.83 / 0.9097$ \\
\hline 25 & $92.78 / 0.8647$ & $\mathbf{9 2 . 7 8 / 0 . 8 6 7 3}$ & $93.10 / 0.8701$ & $93.10 / 0.8701$ \\
\hline \hline Ave. & $93.85 / 0.8789$ & $\mathbf{9 4 . 7 4 / 0 . 9 0 1 9}$ & $96.00 / 0.9240$ & $95.93 / 0.9231$ \\
\hline
\end{tabular}

Table 11: Cross database AU recognition on the structure learnt from MMI database

\begin{tabular}{|l|c|c|c|c|}
\hline $\begin{array}{c}\text { AU in } \\
\text { CK+ }\end{array}$ & $\begin{array}{c}\text { Accuracy (\%) } \\
\text { /F1-score of } \\
\text { single task }\end{array}$ & $\begin{array}{c}\text { Accuracy (\%) } \\
\text { /F1-score of } \\
\text { single task + BN }\end{array}$ & $\begin{array}{c}\text { Accuracy (\%) } \\
\text { /F1-score } \\
\text { of MTL }\end{array}$ & $\begin{array}{c}\text { Accuracy (\%) } \\
\text { /F1-score of } \\
\text { MTL + BN }\end{array}$ \\
\hline 1 & $84.82 / 0.6875$ & $\mathbf{8 4 . 9 9 / 0 . 6 9 2 0}$ & $87.18 / 0.6783$ & $87.02 / \mathbf{0 . 7 6 1 6}$ \\
\hline 2 & $92.75 / 0.7795$ & $92.75 / 0.7795$ & $93.76 / 0.8213$ & $93.42 / 0.8186$ \\
\hline 4 & $80.27 / 0.6139$ & $\mathbf{8 0 . 7 8 / 0 . 6 2 5 0}$ & $73.86 / 0.6301$ & $\mathbf{7 5 . 3 8} / 0.6075$ \\
\hline 5 & $89.88 / 0.6341$ & $\mathbf{9 1 . 2 3 / 0 . 7 1 1 1}$ & $90.73 / 0.6893$ & $\mathbf{9 1 . 0 6 / 0 . 7 3 3 7}$ \\
\hline 7 & $83.98 / 0.3949$ & $82.46 / 0.3882$ & $82.8 / 0.5565$ & $81.28 / 0.5110$ \\
\hline 9 & $89.71 / 0.3297$ & $89.71 / 0.3297$ & $97.30 / 0.8889$ & $97.30 / 0.8889$ \\
\hline 12 & $90.22 / 0.7456$ & $90.22 / 0.7456$ & $90.22 / 0.7852$ & $\mathbf{9 0 . 5 6 / 0 . 7 8 7 9}$ \\
\hline 17 & $87.86 / 0.8182$ & $\mathbf{8 8 . 0 3 / 0 . 8 2 0 3}$ & $85.67 / 0.7826$ & $\mathbf{8 5 . 8 3} / 0.7824$ \\
\hline 25 & $88.53 / 0.8828$ & $88.53 / 0.8828$ & $88.53 / 0.8308$ & $82.97 / 0.8308$ \\
\hline \hline Ave. & $87.56 / 0.6540$ & $\mathbf{8 7 . 6 3 / 0 . 6 6 3 8}$ & $87.78 / 0.7403$ & $87.20 / \mathbf{0 . 7 4 6 9}$ \\
\hline
\end{tabular}

refined by BN structure learned from other database for both databases. For MTL, the performance after improved by BN structure learned from other database is not always improved. Specifically, the learned BN from the MMI database can benefit $\mathrm{AU}$ recognition on the $\mathrm{CK}+$ database in term of F1-score, but not vice versa. Considering that MTL outperforms single task, the results indicate that the structure learning is more effective when the measurements are poor.

It should also be noted that the refinement in AU1 contribute the most of the improvement in recognition, in both single task and MTL. Specifically, the F1score is increased by 0.2125 for single task on the MMI database, and 0.0833 for multi-task on the $\mathrm{CK}+$ database. The strong co-occurrence relationship might be the reason. It is clear that AU2 and AU1 are always linked in the structure learnt from both databases. The dependency tables (Table 6 and Table 8) also correspond to this relationship. Both of the structure and dependency lead to the stability of these BN models learnt from them. 


\subsubsection{Comparison with related work for AU classification}

Although many studies have been done on AU classification, and achieved good performance, only a few work exploits the dependencies among AUs. One representative work is Tong et al. [6], who propose to use a dynamic Bayesian network (DBN) to model the relationships among different AUs and their temporal evolutions for AU classification and conduct the experiments on the CK database. Therefore, it is not exact fair to compare the performance on the CK database with that on the CK+ database. Furthermore, Tong et al.[6] adopted the true skill score as the evaluation metric. True skill score, also called as Hansen Kuiper Discriminant, is the difference between the positive rate and the false positive rate. We adopted $\mathrm{F} 1$-score as the evaluation metric in our paper. Tong et al.[6] used DBN to exploit dynamic patterns in AUs, which is not the focus of our paper. Therefore, we do not compare our work with theirs directly. However, we conduct similar experiment on $\mathrm{CK}+$ database using the learned $\mathrm{BN}$ model to improve the AU recognition from single task. The results are listed in the second column of Table 5. Comparing the fourth and the second column in Table 5, we can find that our proposed method considering AU relations from both labels and features performs better than Tong et al. [6]s' work.

There exist lots of AU classification studies considering each AU individually, which evaluate on the $\mathrm{CK}+$ database. We report the comparison of the proposed method with the current work in Table 5. Comparing the experimental results of our method and those of [4], we find that the accuracies of 8 AUs increase. Furthermore, the average accuracy of our method is larger than that of [4], demonstrating the superiority of our method.

We also compared the results from the most recent research on the MMI database [39] with ours. Our average F1-score is about 5\% higher than that of [39]. Specifically, the F1-scores of 8 AUs increase, which shows the advantage of our approach.

\subsection{Experimental results and analyses for $A U$ intensity estimation}

\subsubsection{Analyses of $A U$ intensity estimation on the McMaster databaseand the DISFA database}

Table 12 provides the AU intensity prediction results on the McMaster database. From Table 12, we can find the following:

First, compared with single task method, AU intensity prediction using MTL increases the average correlation by 0.009 and decreases the average MSE by 0.001, which demonstrates the effectiveness of our method. Using MTL decreases the MSE for almost every AU except for AU4. The results show that our proposed 
Table 12: AU intensity prediction on McMaster database

\begin{tabular}{|c|c|c|c|c|}
\hline AU & $\begin{array}{c}\text { CORR/ICC } \\
\text { /MSE of } \\
\text { single task }\end{array}$ & $\begin{array}{c}\text { CORR/ICC } \\
\text { /MSE of } \\
\text { MTL }\end{array}$ & $\begin{array}{c}\text { CORR/ICC } \\
\text { /MSE of } \\
\text { MTL + BN }\end{array}$ & $\begin{array}{c}\text { CORR } \\
\text { /MSE of } \\
\text { LT-all[23] }\end{array}$ \\
\hline \hline 4 & $0.118 / 0.269 / 0.147$ & $\mathbf{0 . 2 0 1} / \mathbf{0 . 3 0 1} / 0.159$ & $\mathbf{0 . 2 0 2} / \mathbf{0 . 3 0 9} / 0.217$ & $0.03 / 0.51$ \\
\hline 6 & $0.364 / 0.524 / 0.659$ & $\mathbf{0 . 3 6 8} / 0.523 / 0.653$ & $\mathbf{0 . 3 8 6} / \mathbf{0 . 5 4 1} / 0.753$ & $0.60 / 1.06$ \\
\hline 7 & $0.392 / 0.514 / 0.301$ & $\mathbf{0 . 3 9 7} / \mathbf{0 . 5 2 5} / 0.316$ & $\mathbf{0 . 4 7 8 / 0 . 6 4 2 / 0 . 3 6 1}$ & $0.11 / 1.19$ \\
\hline 9 & $0.109 / 0.172 / 0.080$ & $0.103 / 0.156 / \mathbf{0 . 0 7 8}$ & $\mathbf{0 . 1 3 5 / 0 . 2 1 4} / 0.250$ & $0.10 / 0.27$ \\
\hline 10 & $0.257 / 0.392 / 0.069$ & $0.240 / 0.347 / \mathbf{0 . 0 6 8}$ & $\mathbf{0 . 2 9 2} / \mathbf{0 . 4 3 5} / 0.209$ & $0.15 / 0.28$ \\
\hline 12 & $0.381 / 0.561 / 0.800$ & $\mathbf{0 . 3 9 2} / 0.556 / 0.778$ & $0.365 / 0.519 / 0.801$ & $0.60 / 1.12$ \\
\hline 20 & $0.064 / 0.088 / 0.067$ & $\mathbf{0 . 0 7 3} / \mathbf{0 . 0 9 1} / \mathbf{0 . 0 6 5}$ & $\mathbf{0 . 1 0 3} / 0.080 / \mathbf{0 . 0 5 8}$ & $0.09 / 0.19$ \\
\hline 25 & $0.427 / 0.563 / 0.237$ & $\mathbf{0 . 4 2 9} / 0.559 / \mathbf{0 . 2 3 6}$ & $\mathbf{0 . 4 4 7 / 0 . 6 1 5} / 0.338$ & $0.18 / 0.72$ \\
\hline 26 & $0.036 / 0.030 / 0.341$ & $0.031 / \mathbf{0 . 0 3 3} / \mathbf{0 . 3 3 9}$ & $\mathbf{0 . 0 8 5} / 0.024 / \mathbf{0 . 2 8 0}$ & $0.01 / 0.50$ \\
\hline \hline Ave. & $0.239 / 0.346 / 0.300$ & $\mathbf{0 . 2 4 8} / 0.343 / \mathbf{0 . 2 9 9}$ & $\mathbf{0 . 2 7 7} / \mathbf{0 . 3 7 5} / 0.363$ & $0.208 / 0.649$ \\
\hline
\end{tabular}

Table 13: AU intensity prediction on DISFA database

\begin{tabular}{|c|c|c|c|c|}
\hline AU & $\begin{array}{c}\text { CORR/ICC } \\
\text { /MSE of } \\
\text { single task }\end{array}$ & $\begin{array}{c}\text { CORR/ICC } \\
\text { /MSE of } \\
\text { MTL }\end{array}$ & $\begin{array}{c}\text { CORR/ICC } \\
\text { /MSE of } \\
\text { MTL + BN }\end{array}$ & $\begin{array}{c}\text { CORR/ICC } \\
\text { /MSE of } \\
\text { LT-all[23] }\end{array}$ \\
\hline \hline 1 & $0.264 / 0.231 / 3.306$ & $\mathbf{0 . 2 8 3 / 0 . 2 4 9 / 4 . 2 8 0}$ & $0.243 / \mathbf{0 . 2 4 9} / 0.817$ & $0.41 / 0.32 / 0.44$ \\
\hline 2 & $0.513 / 0.428 / 1.506$ & $0.399 / 0.346 / 2.933$ & $\mathbf{0 . 6 8 9} / 0.284 / 1.673$ & $0.44 / 0.37 / 0.39$ \\
\hline 4 & $0.415 / 0.374 / 3.246$ & $\mathbf{0 . 6 5 1 / 0 . 6 3 9 / 2 . 3 5 0}$ & $\mathbf{0 . 7 0 9 / 0 . 8 2 1} / 1.836$ & $0.5 / 0.41 / 0.96$ \\
\hline 5 & $0.239 / 0.167 / 0.332$ & $\mathbf{0 . 2 9 6 / 0 . 2 9 4 / 0 . 4 2 9}$ & $\mathbf{0 . 4 4 5 / 0 . 1 0 4 / 1 . 6 8 6 ~}$ & $0.29 / 0.18 / 0.07$ \\
\hline 6 & $0.462 / 0.451 / 1.838$ & $\mathbf{0 . 4 7 5 / 0 . 4 6 4 / 1 . 8 0 1}$ & $\mathbf{0 . 6 0 0} / 0.229 / 1.563$ & $0.55 / 0.46 / 0.41$ \\
\hline 9 & $0.406 / 0.399 / 2.161$ & $0.385 / 0.381 / 2.344$ & $\mathbf{0 . 4 0 4 / 0 . 4 4 1 / 1 . 8 8 3}$ & $0.32 / 0.23 / 0.31$ \\
\hline 12 & $0.638 / 0.629 / 2.015$ & $0.631 / 0.622 / 2.056$ & $0.460 / \mathbf{0 . 8 6 1} / 0.731$ & $0.76 / 0.73 / 0.4$ \\
\hline 15 & $0.279 / 0.279 / 1.225$ & $0.273 / 0.273 / \mathbf{1 . 2 2 0}$ & $0.127 / \mathbf{0 . 4 5 7} / 0.773$ & $0.11 / 0.07 / 0.17$ \\
\hline 17 & $0.449 / 0.436 / 1.140$ & $\mathbf{0 . 4 4 9 / 0 . 4 3 8 / 1 . 1 2 1}$ & $0.309 / \mathbf{0 . 6 8 9} / 3.066$ & $0.31 / 0.23 / 0.33$ \\
\hline 20 & $0.135 / 0.128 / 1.262$ & $0.122 / 0.114 / 1.272$ & $0.040 / 0.003 / 1.614$ & $0.16 / 0.09 / 0.16$ \\
\hline 25 & $0.680 / 0.679 / 1.466$ & $0.677 / 0.677 / 1.472$ & $0.281 / \mathbf{0 . 8 4 0} / 2.184$ & $0.82 / 0.8 / 0.61$ \\
\hline 26 & $0.139 / 0.132 / 2.782$ & $\mathbf{0 . 1 5 8} / \mathbf{0 . 1 5 2} / \mathbf{2 . 7 0 5}$ & $\mathbf{0 . 2 6 2} / 0.144 / 0.352$ & $0.49 / 0.39 / 0.46$ \\
\hline \hline Ave. & $0.385 / 0.361 / 1.857$ & $\mathbf{0 . 4 0 0 / 0 . 3 8 7} / 1.999$ & $0.381 / \mathbf{0 . 4 2 7} / 1.515$ & $0.43 / 0.36 / 0.39$ \\
\hline
\end{tabular}

multi-task AU intensity estimation with the facial-region grouping strategy can benefit from feature-level AU relations to improve the prediction performance. Specifically, the correlation for AU4 is significantly improved by 0.083 . Furthermore, the performance of its group partner - AU6 are also improved, for the correlation increased by 0.004 and MSE decreased by 0.006 .

Second, comparing the result of our method with the one of MTL, all the AUs except for AU12 have improvement in correlation and the average correlation is increased by 0.033 . Most AUs improve in ICC and the average ICC is increased by 0.032 . It appears that $\mathrm{BN}$ can improve multi-task. Specifically, we can find that AU7 increased in correlation by 0.08 compared with MTL as shown in Table 12. Table 12 also shows that the results refined by BN become worse in the mean square error. From Table 2, we can find the samples in different intensity level in the McMaster database have uneven distribution. Therefore, 
MSE sometimes is not effective for evaluating the performance. For example, if the AU4 is all predicted as 0 , the MSE is 0.145 which is the smallest but the results provide no information. However, this situation will fail in calculating the correlation indicating that the predicted results are meaningless.

Table 13 shows the AU intensity prediction results on the DISFA database. From Table 13, we can find the following:

First, comparing with single task method, AU intensity prediction with MTL increased the average correlation by 0.015 and the average ICC by 0.026. Specifically, the ICC and correlation of almost half AUs are increased by using MTL, and the rest of AUs are nearly the same. This demonstrates that the proposed method using the facial-region grouping procedure can improve the performance by modeling feature-level AU relation. It's worth noting that the correlation for AU4 is greatly improved by 0.236 , and the ICC for AU4 and AU5 is improved by 0.265 and 0.127 respectively. The performance of their group partner, AU6, is also improved with 0.013 improvement in correlation and $\mathrm{ICC}$.

Second, the proposed method outperforms MTL. Specifically, the ICC of half AUs is increased, and thus the average ICC increased by 0.04 . Furthermore, the correlation of half AUs is increased, from 0.019 to 0.29 , and the average MSE is also reduced by $\mathbf{0 . 4 8 4}$. This shows that the label dependencies modeled by BN can successfully improve the correlations between predicted AU intensities and ground-truth.

Fig. 5 and Fig. 6 show the learned BN structure from the McMaster database and the DISFA database respectively. Table 14 and Table 15 list all the dependencies. From the learned BN structure and the dependency table, the label pairs whose conditional probabilities are top ranked or bottom ranked are linked in the $\mathrm{BN}$ in most cases. For example, we can find that $P(A U 6 \mid A U 9)$ is 0.9 in Table 14 indicating that there are strong co-occurrence relationship between AU6 and AU9 and there is link from AU9 to AU6. The learned BN in Fig. 5 modeling this relationship improves the AU6 and AU9 prediction performance by 0.018 and 0.032 in correlation. And we can also find that $P(A U 4 \mid A U 9)$ is $\mathbf{0 . 8 3}$ in Table 15 indicating that there are strong co-occurrence relationship between $\mathrm{AU} 4$ and AU9 and there is a link from AU4 to AU9. The learned BN in Fig. 6 modeling this relationship improves the AU4 and AU9 prediction performance in correlation by 0.058 and 0.019 , and increased ICC by 0.182 and 0.06 respectively. 
Table 14: AU dependencies on McMaster database

\begin{tabular}{|r|l|l|l|l|l|l|l|l|l|}
\hline$\lambda_{i}$ & AU4 & AU6 & AU7 & AU9 & AU10 & AU12 & AU20 & AU25 & AU26 \\
\hline AU4 & 1.00 & 0.58 & 0.30 & 0.18 & 0.16 & 0.41 & 0.03 & 0.24 & 0.07 \\
\hline AU6 & 0.11 & 1.00 & 0.38 & 0.07 & 0.08 & 0.83 & 0.05 & 0.15 & 0.09 \\
\hline AU7 & 0.10 & 0.62 & 1.00 & 0.07 & 0.06 & 0.61 & 0.06 & 0.15 & 0.10 \\
\hline AU9 & 0.45 & 0.90 & 0.56 & 1.00 & 0.39 & 0.57 & 0.05 & 0.46 & 0.07 \\
\hline AU10 & 0.32 & 0.89 & 0.36 & 0.31 & 1.00 & 0.55 & 0.13 & 0.62 & 0.18 \\
\hline AU12 & 0.06 & 0.67 & 0.30 & 0.03 & 0.04 & 1.00 & 0.02 & 0.10 & 0.08 \\
\hline AU20 & 0.04 & 0.42 & 0.28 & 0.03 & 0.10 & 0.20 & 1.00 & 0.40 & 0.19 \\
\hline AU25 & 0.11 & 0.35 & 0.21 & 0.08 & 0.13 & 0.30 & 0.12 & 1.00 & 0.20 \\
\hline AU26 & 0.04 & 0.23 & 0.17 & 0.01 & 0.04 & 0.25 & 0.06 & 0.23 & 1.00 \\
\hline
\end{tabular}

Table 15: AU dependencies on DISFA database

\begin{tabular}{|l|l|l|l|l|l|l|l|l|l|l|l|l|}
\hline$\lambda_{i} \lambda_{j}$ & AU1 & AU2 & AU4 & AU5 & AU6 & AU9 & AU12 & AU15 & AU17 & AU20 & AU25 & AU26 \\
\hline AU1 & 1 & 0.67 & 0.29 & 0.05 & 0.04 & 0.11 & 0.06 & 0.01 & 0.02 & 0.01 & 0.23 & 0.15 \\
\hline AU2 & 0.88 & 1 & 0.23 & 0.08 & 0 & 0.01 & 0.06 & 0 & 0.005 & 0.01 & 0.12 & 0.07 \\
\hline AU4 & 0.20 & 0.12 & 1 & 0.004 & 0.14 & 0.35 & 0.03 & 0.09 & 0.23 & 0.07 & 0.40 & 0.12 \\
\hline AU5 & 0.78 & 0.91 & 0.09 & 1 & 0 & 0 & 0 & 0.03 & 0 & 0.04 & 0 & 0.01 \\
\hline AU6 & 0.04 & 0 & 0.22 & 0 & 1 & 0.21 & 0.64 & 0.03 & 0.01 & 0.09 & 0.81 & 0.06 \\
\hline AU9 & 0.17 & 0.01 & 0.83 & 0 & 0.33 & 1 & 0.01 & 0.11 & 0.04 & 0.09 & 0.56 & 0.10 \\
\hline AU12 & 0.03 & 0.02 & 0.02 & 0 & 0.30 & 0.003 & 1 & 0 & 0 & 0 & 0.95 & 0.04 \\
\hline AU15 & 0.04 & 0 & 0.64 & 0.01 & 0.17 & 0.34 & 0 & 1 & 0.26 & 0.16 & 0.43 & 0.12 \\
\hline AU17 & 0.05 & 0.01 & 0.84 & 0 & 0.01 & 0.06 & 0 & 0.13 & 1 & 0.08 & 0.05 & 0.04 \\
\hline AU20 & 0.05 & 0.05 & 0.62 & 0.02 & 0.51 & 0.33 & 0 & 0.19 & 0.20 & 1 & 0.59 & 0.27 \\
\hline AU25 & 0.08 & 0.03 & 0.19 & 0 & 0.25 & 0.11 & 0.65 & 0.03 & 0.01 & 0.03 & 1. & 0.16 \\
\hline AU26 & 0.24 & 0.09 & 0.26 & 0 & 0.09 & 0.09 & 0.14 & 0.04 & 0.02 & 0.07 & 0.77 & 1 \\
\hline
\end{tabular}

\subsubsection{Comparison with related work for AU intensity estimation}

Among the few studies on AU intensity estimation, three works [21] [22] exploit the dependencies among AUs using DBN [21] or MRF [22] or generative latent tree [23]. The first two works did not report the experimental results on the McMaster database, while the last work conducted experiments on both databases. Therefore, we compare our work with Kaltwang et al.'s [23] . From Table 12, we can find that our proposed method can achieve better performance with higher correlation and lower MSE on the McMaster database, further demonstrating the advantage of our method.

For the DISFA database, from Table 13, we can find the ICCs of our method are higher than those of [23] in most cases. However, the correlation of our method is slightly lower than theirs. These shows our method can 
achieve comparable performance with Kaltwang et al.'s [23]. Since Kaltwang et al. adopted all the images of the DISFA database in their experiments, the number of AUs with lower intensity in their experiments is much larger than ours. Therefore, the MSE may not be an effective metric.

\section{Conclusion}

In this paper, we tackle the problem of AU recognition and AU intensity estimation by exploiting the relations of AUs from both shared features and target labels, which carry crucial top-down and bottom up evidence for improving AU analysis, but has not been thoroughly exploited yet.First, we formulate the AU analyses task into MTL problem with face-region grouping strategy. It is different from the traditional method where all the AUs share the same features space, or none AUs share feature space. Second, we construct the relations among AUs' labels with structural and parameter learning using BN. Finally, we further improve the result of MTL using the learned BN. The results for AU classification and AU intensity estimation show our approach treating AU analyses as a MTL problem outperforms traditional single task methods, and the co-occurrence or exclusive relationship among AUs could also be obtained by $\mathbf{B N}$ and that improves the recognition and estimation results. The cross database $\mathbf{A} \mathbf{U}$ recognition experiments demonstrate the generalization ability of label-level dependency captured by BN.

\section{ACKNOWLEDGMENT}

This work has been supported by the National Science Foundation of China (Grant No. 61175037, 61228304,61473270), and the project from Anhui Science and Technology Agency (1508085SMF223).

\section{References}

[1] Z. Zeng, M. Pantic, G. Roisman, and T. Huang, "A survey of affect recognition methods: Audio, visual, and spontaneous expressions," Pattern Analysis and Machine Intelligence, IEEE Transactions on, vol. 31, no. 1, pp. 39 -58, jan. 2009.

[2] M. Valstar and M. Pantic, "Fully automatic recognition of the temporal phases of facial actions," Systems, Man, and Cybernetics, Part B: Cybernetics, IEEE Transactions on, vol. 42, no. 1, pp. 28 -43, feb. 2012. 
[3] L. van der Maaten and E. Hendriks, "Action unit classification using active appearance models and conditional random fields," Cognitive Processing, vol. 13, pp. 507-518, 2012, 10.1007/s10339-011-0419-7. [Online]. Available: http://dx.doi.org/10.1007/s10339-011-0419-7

[4] G. Littlewort, J. Whitehill, T. Wu, I. Fasel, M. Frank, J. Movellan, and M. Bartlett, "The computer expression recognition toolbox (cert)," in $A u$ tomatic Face Gesture Recognition and Workshops (FG 2011), 2011 IEEE International Conference on, march 2011, pp. 298 -305.

[5] S. Lucey, A. B. Ashraf, and J. F. Cohn, "Investigating spontaneous facial action recognition through aam representations of the face," in In Face Recognition, Delac, 2007, pp. 275-286.

[6] Y. Tong, W. Liao, and Q. Ji, "Inferring facial action units with causal relations," in Computer Vision and Pattern Recognition, 2006 IEEE Computer Society Conference on, vol. 2, 2006, pp. 1623 - 1630.

[7] Y. Tong, J. Chen, and Q. Ji, "A unified probabilistic framework for spontaneous facial action modeling and understanding," Pattern Analysis and Machine Intelligence, IEEE Transactions on, vol. 32, no. 2, pp. 258 -273, feb. 2010.

[8] Y. Li, J. Chen, Y. Zhao, and Q. Ji, "Data-free prior model for facial action unit recognition," Affective Computing, IEEE Transactions on, vol. 4, no. 2, pp. 127-141, April 2013.

[9] Z. Wang, Y. Li, S. Wang, and Q. Ji, "Capturing global semantic relationships for facial action unit recognition," in Computer Vision (ICCV), 2013 IEEE International Conference on, Dec 2013, pp. 3304-3311.

[10] Y. Song, D. McDuff, D. Vasisht, and A. Kapoor, "Exploiting sparsity and co-occurrence structure for action unit recognition," in Automatic Face and Gesture Recognition (FG), 2015 11th IEEE International Conference and Workshops on, vol. 1. IEEE, 2015, pp. 1-8.

[11] X. Zhang and M. Mahoor, "Simultaneous detection of multiple facial action units via hierarchical task structure learning," in Pattern Recognition (ICPR), 2014 22nd International Conference on, Aug 2014, pp. 1863-1868. 
[12] A. Yüce, H. Gao, and J.-P. Thiran, "Discriminant multi-label manifold embedding for facial action unit detection," in Automatic Face and Gesture Recognition (FG), 2015 11th IEEE International Conference and Workshops on, vol. 6. IEEE, 2015, pp. 1-6.

[13] K. Zhao, W.-S. Chu, F. De la Torre Frade, J. Cohn, and H. Zhang, "Joint patch and multi-label learning for facial action unit detection," in IEEE Conference on Computer Vision and Pattern Recognition (CVPR), June 2015.

[14] S. Eleftheriadis, O. Rudovic, and M. Pantic, "Multi-conditional latent variable model for joint facial action unit detection," in International Conference on Computer Vision (ICCV), Santiago, Chile, December 2015.

[15] M. H. Mahoor, S. Cadavid, D. S. Messinger, and J. F. Cohn, "A framework for automated measurement of the intensity of non-posed facial action units," in Computer Vision and Pattern Recognition Workshops, 2009. CVPR Workshops 2009. IEEE Computer Society Conference on. IEEE, 2009, pp. 74 80.

[16] M. S. Bartlett, G. Littlewort, M. Frank, C. Lainscsek, I. Fasel, and J. Movellan, "Fully automatic facial action recognition in spontaneous behavior," in Automatic Face and Gesture Recognition, 2006. FGR 2006. 7th International Conference on. IEEE, 2006, pp. 223-230.

[17] L. Jeni, J. M. Girard, J. F. Cohn, F. De La Torre et al., "Continuous au intensity estimation using localized, sparse facial feature space," in Automatic Face and Gesture Recognition ( $F G), 2013$ 10th IEEE International Conference and Workshops on. IEEE, 2013, pp. 1-7.

[18] A. Savran, B. Sankur, and M. T. Bilge, "Regression-based intensity estimation of facial action units," Image and Vision Computing, vol. 30, no. 10, pp. 774-784, 2012.

[19] S. Kaltwang, O. Rudovic, and M. Pantic, "Continuous pain intensity estimation from facial expressions," in Advances in Visual Computing. Springer, 2012, pp. 368-377.

[20] S. W. Chew, "Recognising facial expressions with noisy data," 2013.

[21] Y. Li, S. M. Mavadati, M. H. Mahoor, and Q. Ji, "A unified probabilistic framework for measuring the intensity of spontaneous facial action units," 
in Automatic Face and Gesture Recognition (FG), 2013 10th IEEE International Conference and Workshops on. IEEE, 2013, pp. 1-7.

[22] G. Sandbach, S. Zafeiriou, and M. Pantic, "Markov random field structures for facial action unit intensity estimation," in Computer Vision Workshops (ICCVW), 2013 IEEE International Conference on. IEEE, 2013, pp. 738745.

[23] S. Kaltwang, S. Todorovic, and M. Pantic, "Latent trees for estimating intensity of facial action units," in Proceedings of the IEEE Conference on Computer Vision and Pattern Recognition, 2015, pp. 296-304.

[24] L. Y. Q. J. Yachen Zhu, Shangfei Wang*, "Multiple-facial action unit recognition by shared feature learning and semantic relation modeling," in the 22nd International Conference on Pattern Recognition, 2014.

[25] P. Wang and Q. Ji, "Multi-view face and eye detection using discriminant features," Computer Vision and Image Understanding, vol. 105, no. 2, pp. 99-111, 2007.

[26] Y. Zhang and Q. Ji, "Active and dynamic information fusion for facial expression understanding from image sequences," Pattern Analysis and Machine Intelligence, IEEE Transactions on, vol. 27, no. 5, pp. 699-714, 2005.

[27] A. Argyriou, T. Evgeniou, and M. Pontil, "Convex multi-task feature learning," Machine Learning, vol. 73, pp. 243-272, 2008, 10.1007/s10994-0075040-8. [Online]. Available: http://dx.doi.org/10.1007/s10994-007-5040-8

[28] Z. Kang, K. Grauman, and F. Sha, "Learning with whom to share in multitask feature learning," in Proceedings of the 28th International Conference on Machine Learning (ICML-11), 2011, pp. 521-528.

[29] G. Schwarz, "Estimating the dimension of a model," The annals of statistics, vol. 6, no. 2, pp. 461-464, 1978.

[30] C. P. de Campos and Q. Ji, "Efficient structure learning of bayesian networks using constraints." Journal of Machine Learning Research, vol. 12, no. 3, pp. 663-689, 2011.

[31] J. Pearl, Probabilistic reasoning in intelligent systems: networks of plausible inference. Morgan Kaufmann, 1988. 
[32] D. Kahle, T. Savitsky, S. Schnelle, and V. Cevher, "Junction tree algorithm," STAT, vol. 631, 2008.

[33] P. Lucey, J. Cohn, T. Kanade, J. Saragih, Z. Ambadar, and I. Matthews, "The extended cohn-kanade dataset (ck+): A complete dataset for action unit and emotion-specified expression," in Computer Vision and Pattern Recognition Workshops (CVPRW), 2010 IEEE Computer Society Conference on, june 2010, pp. $94-101$.

[34] M. Pantic, M. Valstar, R. Rademaker, and L. Maat, "Web-based database for facial expression analysis," in Multimedia and Expo, 2005. ICME 2005. IEEE International Conference on, July 2005, pp. 5 pp.-.

[35] P. Lucey, J. F. Cohn, K. M. Prkachin, P. E. Solomon, and I. Matthews, "Painful data: The unbc-mcmaster shoulder pain expression archive database," in Automatic Face \& Gesture Recognition and Workshops (FG 2011), 2011 IEEE International Conference on. IEEE, 2011, pp. 57-64.

[36] S. Mavadati, M. Mahoor, K. Bartlett, P. Trinh, and J. Cohn, "Disfa: A spontaneous facial action intensity database," Affective Computing, IEEE Transactions on, vol. 4, no. 2, pp. 151-160, 2013.

[37] M. S. Sorower, "A literature survey on algorithms for multi-label learning," Oregon State University, Corvallis, 2010.

[38] P. E. Shrout and J. L. Fleiss, "Intraclass correlations: uses in assessing rater reliability." Psychological bulletin, vol. 86, no. 2, p. 420, 1979.

[39] B. Jiang, M. Valstar, B. Martinez, and M. Pantic, "A dynamic appearance descriptor approach to facial actions temporal modeling," Cybernetics, IEEE Transactions on, vol. 44, no. 2, pp. 161-174, Feb 2014. 


\section{Shangfei Wang}

Shangfei Wang received her BS in Electronic Engineering from Anhui University, Hefei, Anhui, China, in 1996. She received her MS in circuits and systems, and the PhD in signal and information processing from University of Science and Technology of China (USTC), Hefei, Anhui, China, in 1999 and 2002. From 2004 to 2005, she was a postdoctoral research fellow in Kyushu University, Japan. Between 2011 and 2012, Dr. Wang was a visiting scholar at Rensselaer Polytechnic Institute in Troy, NY, USA. She is currently an Associate Professor of School of Computer Science and Technology, USTC. Dr. Wang is an IEEE and ACM member. Her research interests cover computation intelligence, affective computing, and probabilistic graphical models. She has authored or co-authored over 70 publications.

\section{Jiajia Yang}

Jiajia Yang received his BS in Software engineering from Dalian maritime university in 2015, and he is currently pursuing his MS in Computer Science in the University of Science and Technology of China, Hefei, China. His research interesting is affective computing.

\section{Zhen Gao}

Zhen Gao received his BS in computer science from Nanjing University of Science and Technology in 2013, and he is currently pursuing his MS in Computer Science in the University of Science and Technology of China, Hefei, China. His research interesting is affective computing.

\section{Qiang Ji}

Qiang Ji received his PhD in Electrical Engineering from the University of Washington. He is currently a Professor with the Department of Electrical, Computer, and Systems Engineering at Rensselaer Polytechnic Institute (RPI). He recently served as a program director at the National Science Foundation (NSF), where he managed NSF's computer vision and machine learning programs. He also held teaching and research positions with the Beckman Institute at University of Illinois at Urbana-Champaign, the Robotics Institute at Carnegie Mellon University, the Dept. of Computer Science at University of Nevada at Reno, and the US Air Force Research Laboratory. Prof. Ji currently serves as the director of the Intelligent Systems Laboratory (ISL) at RPI.

Prof. Ji's research interests are in computer vision, probabilistic graphical models, information fusion, and their applications in various fields. He has published over 160 papers in peer-reviewed journals and conferences. His research has been supported by major governmental agencies including NSF, NIH, DARPA, ONR, ARO, and AFOSR as well as by major companies including Honda and Boeing. Prof. $\mathrm{Ji}$ is an editor on several related IEEE and international journals and he has served as a general chair, program chair, technical area chair, and program committee member in numerous international conferences/workshops. Prof. Ji is a fellow of IAPR. 